\title{
Berry's phase for a noncyclic rotation of light in a helically wound optical fiber
}

\section{$\operatorname{AUTHOR}(\mathrm{S}):$}

Morinaga, Atsuo; Monma, Akinori; Honda, Kazuhito; Kitano, Masao

\section{CITATION:}

Morinaga, Atsuo ... [et al]. Berry's phase for a noncyclic rotation of light in a helically wound optical fiber. PHYSICAL REVIEW A 2007, 76(5): 052109.

\section{ISSUE DATE:}

2007-11

URL:

http://hdl.handle.net/2433/84620

RIGHT:

(C) 2007 The American Physical Society 
PHYSICAL REVIEW A 76, 052109 (2007)

\title{
Berry's phase for a noncyclic rotation of light in a helically wound optical fiber
}

\author{
Atsuo Morinaga, Akinori Monma, and Kazuhito Honda \\ Department of Physics, Faculty of Science and Technology, 2641 Yamazaki, Noda-shi, Chiba 278-8510, Japan \\ Masao Kitano \\ Department of Electronic Science and Engineering, Kyoto University, Katsura, Nishikyou-ku, Kyoto 615-8510, Japan
}

(Received 18 February 2007; revised manuscript received 23 August 2007; published 16 November 2007)

\begin{abstract}
We investigated Berry's phase for noncyclic evolution using the rotation of a polarization azimuth of linearly polarized light in a partially wound optical fiber over the surface of a cylinder. Using a rotation gauge around the rotation axis, the observed rotation of the polarization azimuth corresponds to the area of a spherical rectangle over the surface enclosed by the contour $C$ of actual evolution, a large circle on the equator, and a longitudinal line connecting them, whereas the rotation of the polar gauge encloses a spherical triangle connecting the zenith of the sphere. The observed values were converted to Berry's phase by transformation from the rotation gauge to the geodesic gauge. Consequently, we could confirm that Berry's phase for a noncyclic evolution is given by the geodesic rule proposed by Samuel and Bhandari.
\end{abstract}

DOI: 10.1103/PhysRevA.76.052109

PACS number(s): 03.65.Vf, 02.40.-k, 42.15.-i, 42.81.Cn

\section{INTRODUCTION}

In 1984, Berry predicted that a quantum system acquires a geometrical phase, in addition to a dynamical phase, if the environment or the Hamiltonian returns to its initial state adiabatically [1]. His idea was that if a spinning particle with a spin eigenvalue $\mathbf{s}$ is in a magnetic field $\mathbf{B}$, which is slowly changed around a circuit on the sphere of direction, then at the end of circuit, the wave function of the spin acquires a geometric phase equal to $s \Omega$, where $\Omega$ is the solid angle subtended by the circuit. This cyclic Berry's phase studied and demonstrated in several physical systems such as polarized light in an optical fiber [2], neutron spins in a helically wound magnetic field [3], the nuclear quadrupole resonance of a rotating crystal [4], and so on [5]. Typically, most of them are classified into two kinds of topological phases. The one is a cycle of changes in the direction of a spinning particle or of propagation of a beam of light (the spinredirection phase), such as Berry's proposal. The other one is a cycle of the SU(2) evolution of the two-state system on the Bloch sphere or polarization of a light on the Poincare sphere (Pancharatnam phase [6]).

Since the discovery of a geometric effect, Berry's phase has been intensively investigated and generalized to nonadiabatic evolution [7]. In 1988, Wu and Li pointed out the effects of quantum adiabatic phase for noncyclic evolution and proposed to test such an effect in muon spin resonance [8]. At the same time, Samuel and Bhandari proposed that Berry's phase appears in a more general context, such as a noncyclic or nonunitary evolution [9]. They pointed out that the geometrical phase shift $\gamma$ between the initial state and the final state can be expressed as a line integral where the contour $C$ is given by the actual evolution from the initial state to the final state and back along any geodesic curve joining the final state to the initial state. In 1990, Weinfurter and Badurek reported the first measurement of Berry's phase for partial cycles with polarized neutrons and observed a linear increase in Berry's phase shift with rotation angle [10]. However, Wagh and Rakhecha indicated that this result was incorrect and proposed correct methods of measuring for a noncyclic SU(2) evolution with an interferometry [11] or with a polarimetry [12]. In a noncyclic SU(2) evolution, amplitude and phase of interference signal must be determined under an additive dynamical phase. Indeed, they demonstrated noncyclic phases experimentally using neutron interferometer [13], although it was followed by a comment and reply $[14,15]$. Recently, Filipp et al. have succeeded to measure the noncyclic phase due to spatial evolution in a neutron interferometer [16] and Klepp et al. have measured for mixed state $\mathrm{SU}(2)$ evolution in neutron polarimetry [17]. Like this, the noncyclic $\mathrm{SU}(2)$ evolution has been investigated experimentally without ambiguity. However, the noncyclic evolution for the spin-redirection phase has not yet been verified experimentally, except for the case of a half cycle [18].

\section{NONCYCLIC SPIN-REDIRECTION PHASE IN OPTICS}

A number of manifestations of the geometric phase occur in optics and was summarized in review papers by Bhandari [19], Hariharan [20], and so on. In optics, there are also two types of Berry's phase. The spin-redirection phase in photon was first found by Chiao et al. skillfully by rotating the propagation direction of the photon in 1986 [2,21]. They examined a linearly polarized laser beam injected into a single helically wound optical fiber on a cylinder with a uniform pitch angle $\theta$ of the helix. The continuum trace of the photon in the fiber is converted to a rotation of the propagation direction of the light over a cone of angle $\theta$ about the polar axis. The linearly polarized beam is composed of the right-handed circularly polarized light and the left-handed circularly polarized light. After the direction of the optical beam is rotated by a whole turn, Berry's phase for righthanded circularly polarized light is given by

$$
\gamma_{+}=-2 \pi(1-\cos \theta)=-\Omega(C),
$$

while that for left-handed circularly polarized light is given by $\gamma_{-}=-\gamma_{+}$, whose size corresponds to the solid angle $\Omega$ subtended by the direction on the sphere of direction of light. 
Therefore, the resultant polarization direction of light is rotated by $\gamma+$. The propagation directions of the input and output of the fiber were kept identical and the polarization direction was measured at the input and output points using the same gauge, which was transported parallel from the initial point to the final point. They verified that the measured angle of rotation of linearly polarized light is in a good agreement with the solid angle in the momentum space.

Using a linearly polarized light system, it is well known that Berry's phase can be measured free from dynamical phase shift $[21,22]$. Here, we shall treat Berry's phase for noncyclic rotation of the propagation direction of the photon. Figure 1 shows a map of the propagation direction of light rotating over a cone of polar angle $\theta$ for the partial angle $\phi$ on a sphere of propagation direction. The phase shift $\Theta$ observed between the initial and the final states differs depending on the gauge, i.e., $\Theta=\gamma-\beta_{G}$, where $\beta_{G}$ is a parameter depending on the gauge. However, Samuel and Bhandari pointed out that the geometrical phase shift $\gamma$ between the initial state and the final state can be expressed as a line integral where the contour $C$ is given by the actual evolution from the initial state to the final state and back along any geodesic curve joining the final state to the initial state [9].

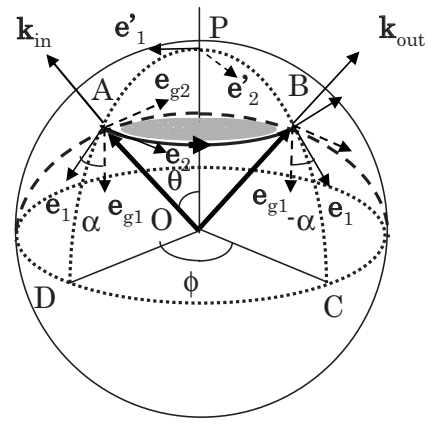

FIG. 1. Sphere of the propagation direction of the light $\mathbf{k}$. $\mathbf{k}$ rotates from $\mathbf{k}_{\text {in }}(A)$ to $\mathbf{k}_{\text {out }}(B)$ with a fixed angle $\theta$ about polar axis during a partial angle $\phi . P$ is the zenith of the sphere. $C$ and $D$ are cross points where longitude intersects the equator. $\mathbf{e}_{1}$ and $\mathbf{e}_{2}$ are two basis vectors of the rotation gauge. $\mathbf{e}_{1}^{\prime}$ and $\mathbf{e}_{2}^{\prime}$ are those of the polar gauge. $\mathbf{e}_{g 1}$ and $\mathbf{e}_{g 2}$ are those of the geodesic gauge.

Then, $\Theta$ is clearly a gauge-invariant quantity and $\gamma$ is measurable. Thus, the solid angle can be expressed by the area of the foliage, which is enclosed by the locus of rotation and the geodesic rule. Consequently, Berry's phase is given by

$$
\gamma(\theta, \phi)=-m \begin{cases}2 \sin ^{-1}\left(\frac{\sin (\phi / 2)}{\sqrt{1+\tan ^{2} \theta \cos ^{2}(\phi / 2)}}\right)-\phi \cos \theta, & 0 \leq \phi \leq \pi \\ 2\left[\pi-\sin ^{-1}\left(\frac{\sin (\phi / 2)}{\sqrt{1+\tan ^{2} \theta \cos ^{2}(\phi / 2)}}\right)\right]-\phi \cos \theta, & \pi \leq \phi \leq 2 \pi .\end{cases}
$$

However, it will be difficult experimentally to define geodesic gauges on the cylinder at the initial and final points of the fiber in order to measure the change in polarization direction. Kwon et al. measured only Berry's phase for a half-cycle by use of a half-turn optical fiber [18]. They defined the solid angle for a half-cycle path as an area subtended by connecting the initial and the final points by a great circle passing through the pole. They confirmed experimentally this phase change, but there was no definite description on their gauge used to measure the rotation of the polarization direction in their paper.

Recently, we measured the spin-redirection phase for atoms with a magnetic quantum number of $m$ using a polarized atom interferometer, but the observed phase shift was in proportion to the rotation angle of the magnetic field [23]. In the present study, we aimed to confirm the geodesic rule for a partial turn pointed out by Samuel and Bhandari [9], using polarized light in a single-mode fiber system similar to that used by Chiao et al. [2], except that the fiber was wound with an angle $\theta$ of the helix on a cylinder for a partial turn of a rotation angle $\phi$. To measure the polarization azimuth of linearly polarized light as a geodesic rule, our strategy is to use a conventional rotating gauge about the rotation axis or a polar gauge fixed on the point of pole of the rotation axis, instead of using the geodesic gauge on a cylinder. The observed changes in polarization direction, which are dependent on the gauge, were converted to the phase shift measured using a geodesic gauge. Then, the noncyclic spinredirection phase was obtained indirectly.

\section{DEFINITION OF GAUGE}

In order to measure the rotation angle of linear polarization for a partial cycle, the frame of reference for each end of the fiber has to be fixed beforehand. In Fig. 1, we assume that a unit vector for the propagation direction of the light is $\mathbf{k}$. In general, for each $\mathbf{k}$, we must define two basis vectors $\mathbf{e}_{1}(\mathbf{k})$ and $\mathbf{e}_{2}(\mathbf{k})$, which are orthogonal to each other and to $\mathbf{k}$. These basis vectors can be used to define the direction of polarization at each end. We assume that the polarization of the incident light beam $\mathbf{k}_{\text {in }}$ is $\mathbf{e}_{\text {in }}=\mathbf{e}_{1}\left(\mathbf{k}_{\text {in }}\right)$. The polarization vector of the output beam $\mathbf{k}_{\text {out }}$ is represented as

$$
\mathbf{e}_{\text {out }}=(\cos \Theta) \mathbf{e}_{1}\left(\mathbf{k}_{\text {out }}\right)+(\sin \Theta) \mathbf{e}_{2}\left(\mathbf{k}_{\text {out }}\right),
$$

where the rotation angle between vectors $\mathbf{e}_{\text {out }}$ and $\mathbf{e}_{1}\left(\mathbf{k}_{\text {out }}\right)$ is $\Theta$. Note that the rotation angle depends on the choice of the frame of reference except in the case of a closed cycle, where the input and output frames coincide. 


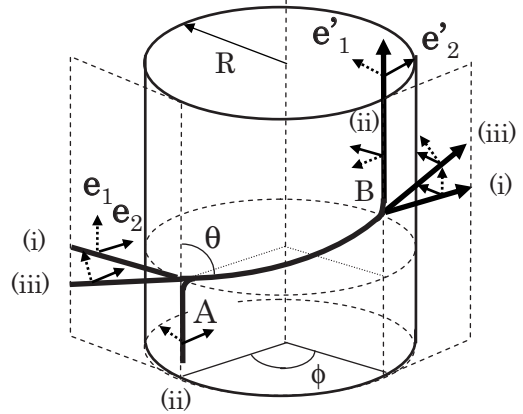

FIG. 2. Experimental setup. A single-mode fiber was wound on the cylinder from $A$ to $B$ with a uniform helix $\theta$ for angle of $\phi$. Before the input and after the output, the direction of the fiber in the tangential plane is (i) $\theta_{0}=\pi / 2$, (ii) $\theta_{0}=0$, and (iii) $\theta_{0}=\theta$. $\mathbf{e}_{1}$ and $\mathbf{e}_{2}$ are two basis vectors of the rotation gauge. $\mathbf{e}_{1}^{\prime}$ and $\mathbf{e}_{2}^{\prime}$ are those of the polar gauge.

We assume $|\mathbf{k}|=1$ and represent it using a polar coordinate system such as $\mathbf{k}=(\theta, \phi)$. A straight way of assigning basis vectors is

$$
\mathbf{e}_{1}(\mathbf{k})=\frac{\partial}{\partial \theta} \mathbf{k}, \quad \mathbf{e}_{2}(\mathbf{k})=\frac{1}{\sin \theta} \frac{\partial}{\partial \phi} \mathbf{k} .
$$

We call this assignment the "rotating gauge." We can also define another gauge called the "polar gauge" as

$$
\begin{aligned}
& \mathbf{e}_{1}^{\prime}(\mathbf{k})=(\cos \phi) \mathbf{e}_{1}(\mathbf{k})-(\sin \phi) \mathbf{e}_{2}(\mathbf{k}), \\
& \mathbf{e}_{2}^{\prime}(\mathbf{k})=(\sin \phi) \mathbf{e}_{1}(\mathbf{k})-(\cos \phi) \mathbf{e}_{2}(\mathbf{k}) .
\end{aligned}
$$

\section{EXPERIMENT}

The experimental setup is schematically shown in Fig. 2 . A conventional single-mode fiber was wound smoothly on the cylinder with a uniform helix $\theta$, which is the angle between the axis of the local fiber and the axis of the helix. The radius of the cylinder is $8.8 \mathrm{~cm}$. Suppose that the cylinder is placed vertically. The fiber enters the cylinder at the initial point $A$ from a tangential direction to the surface of the cylinder, is wound for an angle $\phi$ projected on a horizontal cross section of the cylinder (corresponding to an equator plane in the sphere of the propagation direction), and exits the cylinder in a tangential direction at the final point $B$, as shown in Fig. 2. A He-Ne laser with linearly polarized light was used to measure the rotation of the polarization in the wound fiber. The inclinations of the fiber at the input and output points, $\theta_{0}$ were kept identical in their tangential planes.

The following three cases were studied: (i) $\theta_{0}=\pi / 2$; perpendicular to the rotation axis (equator plane), (ii) $\theta_{0}=0$; parallel to the rotation axis, and (iii) $\theta_{0}=\theta$; a straight line. Except for case (ii), the coordinate used to measure the polarization azimuth cannot be transported in a straight line from $A$ to $B$ without rotation around the rotation axis. Therefore, a simple rotation gauge was used. The $\mathbf{e}_{3}$ axis of the gauge used was defined to be the direction of the propagation

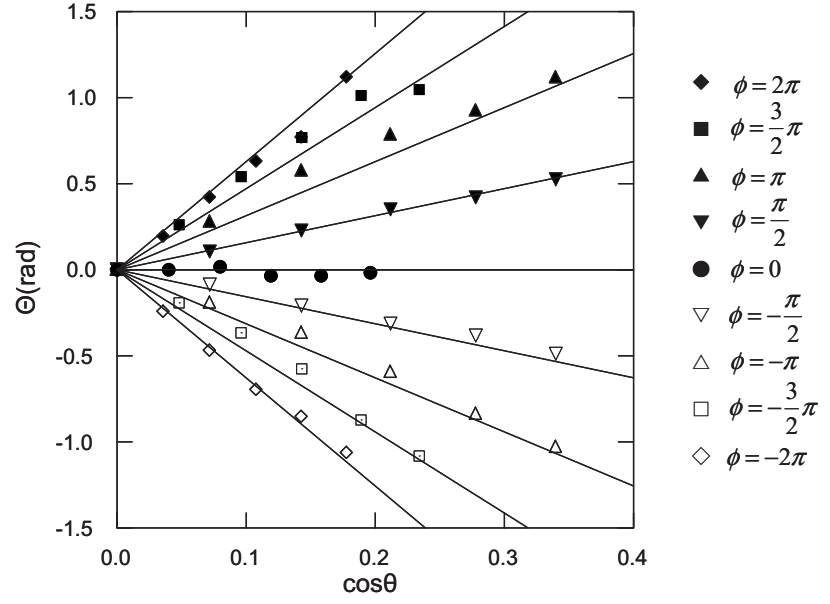

FIG. 3. Rotation angle of the polarization azimuth of the fiber with $\theta_{0}=\pi / 2$ measured by a rotation gauge as a function of $\cos \theta$ for various $\phi$. Uncertainty of each data is almost the same as the symbol size. Solid lines show $\Theta=\phi \cos \theta$.

of the light, i.e., the direction of the fiber, at the initial or final point, the $\mathbf{e}_{1}$ axis is in the tangential plane and the $\mathbf{e}_{2}$-axis was defined to be the line connecting the initial or final point to the center of the cylinder on the horizontal plane. At the initial point, the polarization azimuth of the linearly polarized light was set to be the $\mathbf{e}_{1}$ axis. The fiber was wound in a right-handed $(\phi>0)$ or left-handed $(\phi<0)$ direction as it moved to the top of the cylinder. Then, the rotation angle of the polarization azimuth $\Theta$ was assumed to be positive when it rotated from the $\mathbf{e}_{1}$ axis to the $\mathbf{e}_{2}$ axis at the final point and was measured with an uncertainty of about $\pm 0.05 \mathrm{rad}$. Before the measurement of the partial rotation, we examined the cyclic evolution of a whole turn and ascertained that the measured rotation angle of the polarization azimuth was consistent within $3 \%$ of the solid angle of $2 \pi(1-\cos \theta)$.

\section{RESULTS AND DISCUSSION}

Figure 3 shows the rotation angle of the polarization azimuths versus $\cos \theta$ measured for case (i). Except for the rotation on a large circle of the globe $(\theta=\pi / 2)$, the polarization azimuth varies linearly with $\cos \theta$, and its absolute slope increases with the rotation angle $\phi$. These results could be described accurately by straight lines of the form $\Theta$ $=\phi \cos \theta$ (solid lines). This fact can be explained by assuming that the rotation gauge encloses a spherical rectangle $A B C D$ on the spherical surface in the propagation direction (see Fig. 1), whose solid angle equals $\phi \cos \theta$ if $C$ and $D$ are the points where longitudes through $A$ and $B$ intersect the equator of the direction sphere, respectively. These operations are carried out by smoothly bending the fiber from $\theta$ to $\pi / 2$ at $A$ or $B$. Finally, the coordinates form a closed loop. Furthermore, the experimental results of cases (ii) and (iii) were the same as those of case (i) when we used the same gauge. These results mean that the rotation angle of the polarization azimuth obtained by the rotation gauge is the area of a spherical rectangle $A B C D$ whether or not the coordinates 


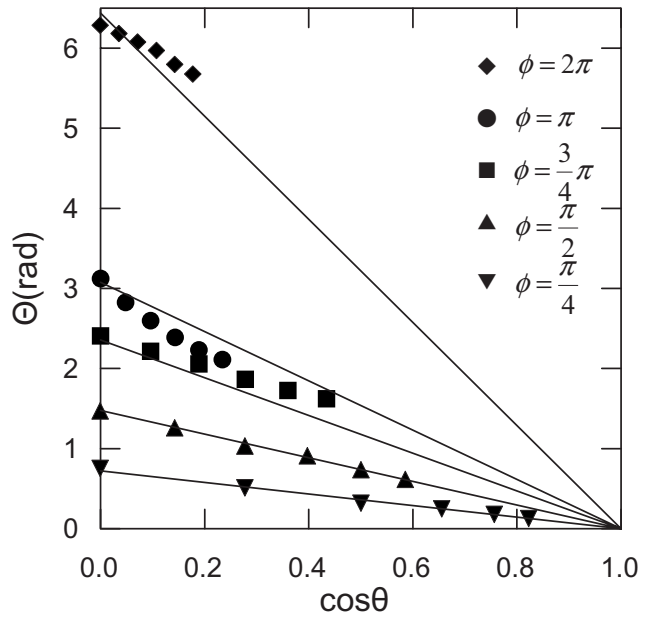

FIG. 4. Rotation angle of the polarization azimuth of the fiber with $\theta_{0}=0$ measured by a polar gauge as a function of $\cos \theta$ for various values of $\phi<0$. Uncertainty of each data is almost the same as the symbol size. Solid lines show $\Theta=-\phi(1-\cos \theta)$.

bend at $A$ or $B$. Thus, we conclude that the rotation gauge measures the area of the spherical rectangle enclosed by the actual evolution from the initial state to the final state and back along a large circle of the equator joining the final and initial states.

In the case of (ii), we measured the rotation of the polarization azimuth using the polar gauge. The coordinates at the initial point were defined as before, but the coordinates were transported parallel to the final point, so that the directions of the three axes were preserved in this case. The results measured using this gauge are shown in Fig. 4. The number of observed rotations of the polarization azimuth for various values of $\phi<0$ decreases with $\cos \theta$ and converges to zero at $\cos \theta=1$. This behavior is accurately well described by lines $\gamma=-\phi(1-\cos \theta)$, which are shown in Fig. 4 as solid lines. This fact shows that this polar gauge encloses a spherical triangle $A B P$ on the sphere, where $P$ denotes the zenith of the sphere. We can easily understand that the present gauge is the fixed gauge at $P$ or at the center of the sphere. At the final point, the $\mathbf{e}_{1}$ axis of the rotation gauge has rotated $\phi$ from that of the fixed gauge. Then, the observed rotation angle of the polarization azimuth has to be converted from $\phi \cos \theta$ to $-\phi(1-\cos \theta)$ by subtracting $\phi$. The results in Figs. 3 and 4 verify this gauge transformation.

Finally, we try to transform the rotation gauge to the geodesic gauge. The coordinates of the rotation gauge defined on the cylinder in case (iii) are given in the sphere of the light direction, together with coordinates at the initial and final positions of the geodesic gauge, as shown in Fig. 1. At the initial point, the $\mathbf{e}_{3}$ axes of both gauges are in the same direction, but the $\mathbf{e}_{1}$ and $\mathbf{e}_{2}$ axes of the rotation gauge are different from the $\mathbf{e}_{g 1}$ and $\mathbf{e}_{g 2}$ axes of the geodesic gauge. We assume that the $\mathbf{e}_{g 1}$ axis rotates by $\alpha$ from the $\mathbf{e}_{1}$ axis. Similarly, the $\mathbf{e}_{1}$ axis rotates by $\beta$ from the $\mathbf{e}_{g 1}$ axis of the geodesic gauge at the final point. The $\mathbf{e}_{g 1}$ or $\mathbf{e}_{g 2}$ axis of the geodesic gauge must be preserved in the plane formed by the geodesic line and the center of the sphere. Here, we assumed it was the $\mathbf{e}_{g 2}$ axis. Then, the rotation of the polarization
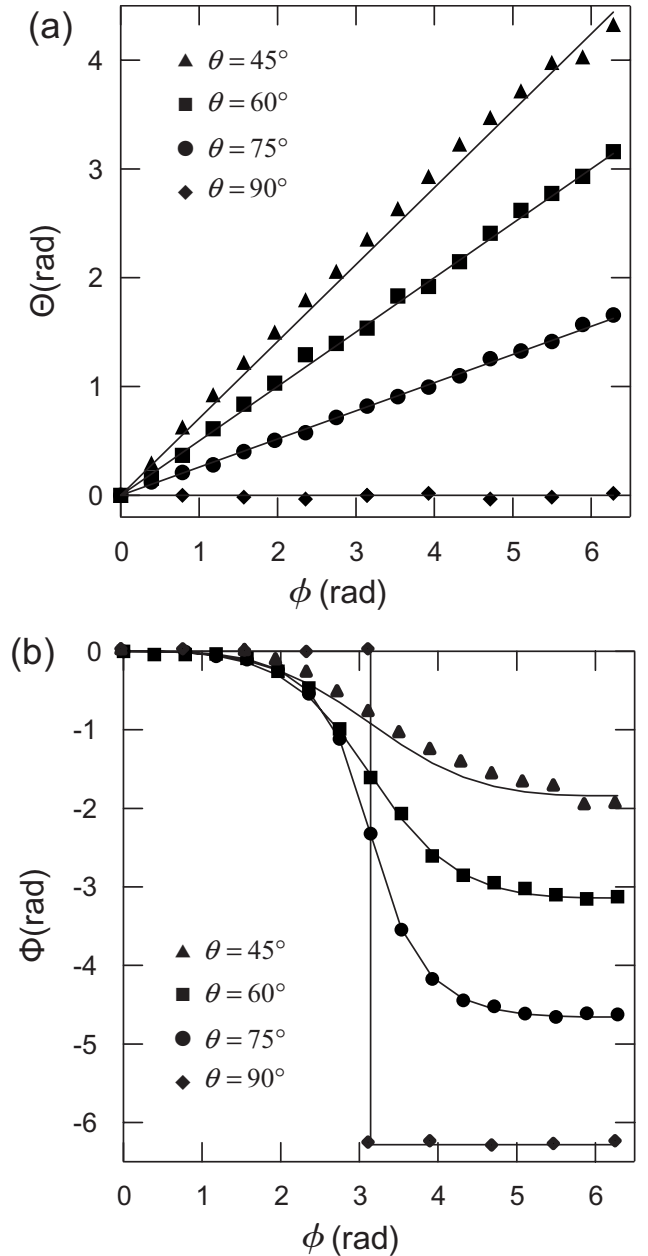

FIG. 5. (a) Rotation angle of the polarization azimuth of the fiber with $\theta_{0}=\theta$ measured by a rotation gauge as a function of $\phi$. Uncertainty of each data is almost the same as the symbol size. Solid lines show $\Theta=\phi \cos \theta$. (b) Rotation of the polarization azimuth converted from values of (a) to those of geodesic gauge. Solid lines show theoretical values.

azimuth on the geodesic gauge $\Phi$ is given by

$$
\Phi=\Theta-\alpha+\beta .
$$

If we measure the input and the final points from the center of the rotation, then

$$
\alpha=\arg \left(\frac{\cos (\phi / 2)}{\cos \theta}+i \sin (\phi / 2)\right),
$$

and $\beta=-\alpha$. Finally, we obtain

$$
\Phi=\Theta-2 \arg \left(\frac{\cos (\phi / 2)}{\cos \theta}+i \sin (\phi / 2)\right) .
$$

The measured $\Theta$ can be converted to $\Phi$ using the above formula. Figure 5(a) shows $\Theta$ in case (iii) as a function of $\phi$, and in Fig. 5(b), $\Phi$ converted from $\Theta$ in Fig. 5(a), together with the theoretical values of the geodesic rule. The converted values are in good agreement with the theoretical 
values. Consequently, we could demonstrate that Berry's phase for a partial cycle for any angle of $\theta$ is related to the area of the foliage enclosed by the locus of rotation and the geodesic rule, which was predicted by Samuel and Bhandari [9]. For rotation angles larger than $\pi, 2 \pi$ is added to $\Phi$ for $\theta=\pi / 2$. As the geodesic line is not defined solitary at $\theta$ $=\pi / 2$ for a half-cycle $(\phi=\pi)$, Berry's phase is not also specified. The present results for a half-cycle except for $\theta$ $=\pi / 2$ are coincident to the result $-\pi(1-\cos \theta)$, which was observed by Kwon et al. [18]. It should be noted that the phase shift for a half-cycle path is the same as that measured by a polar gauge.

In the above experiment, the pole and the equator of the direction sphere are defined uniquely related to the rotation axis of the propagation direction of the light. However, generally, one can define a pole and an equator arbitrarily on the sphere, so that the rotation angle of the polarization azimuth measured by a polar gauge or a rotation gauge is different depending on the defined pole and equator. Contradiction, the rotation angle measured by the geodesic gauge is an invariable quantity, since there is only one geodesic line connecting the initial point and the final point.

\section{CONCLUSION}

In summary, we investigated Berry's phase for noncyclic evolution using the rotation of a polarization azimuth of a linearly polarized light in a partially wound optical fiber on a cylinder. Using a rotation gauge around the rotation axis, the observed rotation of the polarization azimuth corresponds to the area of a spherical rectangle over the surface enclosed by the contour $C$ of actual evolution, a large circle on the equator, and a longitudinal line connecting them. The observed values were converted to the Berry's phase by a transformation from the used gauge to the geodesic gauge. Consequently, we could demonstrate that Berry's phase for a partial evolution is given by the geodesic rule proposed by Samuel and Bhandari [9]. Also, we could confirm that the observed phase shift will be proportional to the rotation angle $\phi$, as obtained in the experiment using an atom interferometer if a polar gauge on the zenith is used [23].

\section{ACKNOWLEDGMENT}

We thank H. Uesugi for her assistance in the course of the experiment.
[1] M. V. Berry, Proc. R. Soc. London, Ser. A 392, 451 (1984).

[2] A. Tomita and R. Y. Chiao, Phys. Rev. Lett. 57, 937 (1986).

[3] T. Bitter and D. Dubbers, Phys. Rev. Lett. 59, 251 (1987).

[4] R. Tycko, Phys. Rev. Lett. 58, 2281 (1987).

[5] Geometric Phases in Physics, edited by A. Shapere and F. Wilczek (World Scientific, Singapore, 1989).

[6] S. Pancharatnum, Proc. Indian Acad. Sci., Sect. A 44, 247 (1956).

[7] Y. Aharonov and J. Anandan, Phys. Rev. Lett. 58, 1593 (1987).

[8] Y.-S. Wu and H.-Z. Li, Phys. Rev. B 38, 11907 (1988).

[9] J. Samuel and R. Bhandari, Phys. Rev. Lett. 60, 2339 (1988).

[10] H. Weinfurter and G. Badurek, Phys. Rev. Lett. 64, 1318 (1990).

[11] A. G. Wagh and V. C. Rakhecha, Phys. Lett. A 197, 107 (1995).

[12] A. G. Wagh and V. C. Rakhecha, Phys. Lett. A 197, 112 (1995).
[13] A. G. Wagh, V. C. Rakhecha, P. Fischer, and A. Ioffe, Phys. Rev. Lett. 81, 1992 (1998).

[14] R. Bhandari, Phys. Rev. Lett. 83, 2089 (1999).

[15] A. G. Wagh, V. C. Rakhecha, P. Fischer, and A. Ioffe, Phys. Rev. Lett. 83, 2090 (1999).

[16] S. Filipp, Y. Hasegawa, R. Loidl, and H. Rauch, Phys. Rev. A 72, 021602(R) (2005); S. Filipp, Y. Hasegawa, R. Loidl, and H. Rauch, J. Res. Natl. Inst. Stand. Technol. 110, 251 (2005).

[17] J. Klepp, S. Sponar, Y. Hasegawa, E. Jericha, and G. Badurek, Phys. Lett. A 342, 48 (2005).

[18] O. J. Kwon, H. T. Lee, S. B. Lee, and S. S. Choi, Opt. Lett. 16, 223 (1991).

[19] R. Bhandari, Phys. Rep. 281, 1 (1997).

[20] P. Hariharan, Prog. Opt. 48, 149 (2005).

[21] R. Y. Chiao and Y. S. Wu, Phys. Rev. Lett. 57, 933 (1986).

[22] R. Bhandari, Phys. Lett. A 138, 469 (1989).

[23] A. Morinaga, T. Aoki, and M. Yasuhara, Phys. Rev. A 71, 054101 (2005) 